\title{
Autonomous Chances and the Conflicts Problem
}

\author{
Christopher J. G. Meacham \\ Forthcoming in Asymmetries in Chance and Time, ed. Handfield and Wilson.
}

\begin{abstract}
In recent work, Callender and Cohen (2009) and Hoefer (2007) have proposed variants of the account of chance proposed by Lewis (1994). One of the ways in which these accounts diverge from Lewis's is that they allow special sciences and the macroscopic realm to have chances that are autonomous from those of physics and the microscopic realm. A worry for these proposals is that autonomous chances may place incompatible constraints on rational belief. I examine this worry, and attempt to determine (i) what kinds of conflicts would be problematic, and (ii) whether these proposals lead to problematic conflicts. After working through a pair of cases, I conclude that these proposals do give rise to problematic conflicts.
\end{abstract}

\section{Introduction}

David Lewis (1994) proposed an influential regularity account of laws and chances. Roughly, the idea was that the laws and chances are given by the description of the occurrent facts which best satisfies the competing desiderata of simplicity and informativeness. ${ }^{1}$ Of course, our assessment of how simple a given description is will depend on the language we use to express the description. So Lewis required us to employ a special language when evaluating these descriptions-a language whose predicates correspond to the perfectly natural properties. ${ }^{2}$

On Lewis's account there is, at each world, a single, unified collection of laws and chancesthe collection that offers, on balance, the simplest and most informative description of the occurrent facts. Callender and Cohen (2009) have complained that this only gives us the laws and chances of something like fundamental physics. What about the laws and chances of special sciences, like biology or economics? If we think we can derive these special science laws from those of physics, then this needn't bother us that much. But Callender and Cohen (2010) have argued that special science laws and chances can be autonomous from those of physics. And Lewis's account of laws and chances doesn't seem to allow room for that.

${ }^{1}$ I'm glossing over a number of details here; see Lewis (1994) for a more detailed characterization of his account. In addition to simplicity and informativeness, Lewis also invokes a third desiderata, "fit", which is the chance assigned to the actual world history by the chances of the candidate description. To simplify things, I'm lumping considerations of fit in with informativeness.

${ }^{2}$ See Lewis (1983). 
In a similar vein, Hoefer (2007) has argued that an adequate account of chance should be able to recover the chances of ordinary life, like the chance of rain or of this coin landing heads, no matter what the laws and chances of fundamental physics are. If so, then we need an account of chance that doesn't depend on what fundamental physics turns out to be.

In light of these considerations, Callender and Cohen (2009) and Hoefer (2007) have proposed modified versions of Lewis's regularity account of laws and chance. ${ }^{3}$ These modified accounts allow for multiple autonomous chance theories. ${ }^{4}$ But a natural worry arises for accounts like these. Chances are generally taken to place constraints on rational belief. All else being equal, if you know the chance of some event is $1 / 2$, then your credence in that event should be $1 / 2$. But if we have multiple autonomous chance theories, it seems like these different chance theories could impose conflicting constraints on rational belief. Call this the Conflicts Problem.

Callender and Cohen (2009) and Hoefer (2007) both discuss the Conflicts Problem, and argue that this worry does not pose a problem for their respective accounts. In this paper I will explore whether they are right.

In the next section, I will sketch some background on chance and chance-credence principles, and examine which kinds of chancy conflicts are problematic. In the third section, I discuss Callender and Cohen's account, and explore whether this account leads to problematic conflicts. In the fourth section, I sketch Hoefer's account, and explore whether his account leads to problematic conflicts. In the fifth section, I'll consider some possible replies to these worries. In the sixth section, I conclude by briefly assessing the moral of these investigations.

\section{Background}

\subsection{Chance-Credence Principles}

To begin, let us assume that rational agents are Bayesian-they have probabilistic credences and update via conditionalization. ${ }^{5}$

The canonical account of the relation between credence and chance is given by Lewis's Principal Principle. ${ }^{6}$ Let $i c$ be a rational initial credence function, let $T$ be a proposition stating the complete chance theory of a world, and let $H$ be a proposition stating the complete history up to a time at that world. Then for an arbitrary proposition $A$, Lewis's principle imposes the

\footnotetext{
${ }^{3}$ Though these are not their only motivations for revising Lewis's account. For example, the accounts offered by Callender and Cohen (2009) and Hoefer (2007) don't have to appeal to the distinction between natural and non-natural properties.

${ }^{4}$ Others have also suggested modified versions of Lewis's account of laws and chances in a similar vein; for example, see Taylor (1993), Schrenk (2008), Glynn (2010) and Dunn (2011). Whether these views are subject to similar worries depends on how the details of these accounts are spelled out.

Note that the Conflicts Problem does not arise from just allowing the probabilities given in ordinary life or in the special sciences to be chances. The account proposed by Handfield and Wilson (2012) allows for such chances, for example, and is unproblematic. What raises the Conflicts Problem is allowing these chances to be autonomous.

${ }^{5} \mathrm{An}$ agent with credences $c r$ satisfies conditionalization iff, whenever she receives some new evidence $E$, her new credences $c r_{E}$ are such that $c r_{E}(A)=\operatorname{cr}(A \mid E)$, if defined.

${ }^{6}$ See Lewis (1986).
} 
following constraint: ${ }^{7}$

$$
i c(A \mid T H)=c h_{T H}(A), \text { if defined. }
$$

That is, a rational initial credence in $A$, conditional on a complete chance theory $T$ and a complete history up to a time $H$, is equal to the chance assigned to $A$ by the chance distribution picked out by $T$ and $H .^{8}$

Lewis formulated this principle with dynamical chances in mind-chances describing how likely it is for a world in one state at one time to transition into another state at a later time. As a consequence, Lewis took a chance distribution to be picked out by a complete chance theory $T$ and a complete history up to a time, $H$. In what follows, I'll replace this complete history up to a time $H$ with a background-information-specifying proposition $K$. This allows us to accommodate a wider variety of chance theories, like those of statistical mechanics and the special sciences. ${ }^{9}$ So the version of the Principal Principle I'll employ is this:

$$
i c(A \mid T K)=\operatorname{ch}_{T K}(A), \text { if defined. }
$$

Although Lewis's Principal Principle does a good job of capturing the intuitive relationship between credence and chance, it is problematic for proponents of regularity accounts of chance. In particular, the Principal Principle entails that $c h_{T K}$ must assign $T$ and $K$ a chance of 1 :

$$
c h_{T K}(T K)=i c(T K \mid T K)=1 .
$$

But while proponents of regularity accounts accept that $c h_{T K}(K)=1$, they'll generally deny that $c h_{T K}(T)=1$.

To see why, consider a simple regularity account, on which the chance of a coin landing heads equals the proportion of coin tosses that have landed heads, where these events are treated as independent and identically distributed. ${ }^{10}$ Suppose there are only two coin tosses, one of which lands heads. Then according to this simple regularity account, the resulting chance theory $T$ will assign a chance of $1 / 2$ to heads, and a chance of $1 / 4$ to both coins landing heads. But note

\footnotetext{
${ }^{7}$ Where I'm employing to convention of using concatonated proposition names (e.g., $A B$ ) to stand for the conjunction of those propositions $(A \wedge B)$.

${ }^{8}$ Although a lot of discussion regarding the Principal Principle has been devoted to the notion of admissibility, Lewis's second formulation of the Principal Principle, (1), has no admissibility clause (see Lewis (1986)). It is only Lewis's first formulation of the Principal Principle:

$$
i c\left(A \mid\left\langle c h_{t}(A)=x\right\rangle E\right)=x \text {, if } E \text { is admissible with respect to }\left\langle c h_{t}(A)=x\right\rangle,
$$

that has an admissibility clause. This admissibility clause is required in order to make the two formulations equivalent; without the admissibility clause, Lewis's first formulation of the Principal Principle is strictly weaker than the second (see Meacham (2010), section 4).

For a discussion of whether one could appeal to admissibility in order to escape the Conflicts Problem, see section 5.

${ }^{9}$ Although Lewis himself would have resisted this extension of the Principal Principle to theories like statistical mechanics, Callender and Cohen (2009) and Hoefer (2007) do not. So with respect to this debate, this is common ground.

${ }^{10} \mathrm{By}$ abandoning the latter assumption one can escape these worries, though this leads to other difficulties; see Arntzenius and Hall (2003).
} 
that if both coins were to land heads, then a different chance theory $T^{*}$ would obtain-since all of the coin tosses land heads, the chance of heads would be 1 . So the chance theory $T$ is in a sense self-undermining - it assigns a positive chance to some other chance theory $T^{*}$ obtaining. Likewise, since $T$ only obtains if one of the two coin tosses lands heads, it assigns itself a chance of less than 1.

As we saw from (3) above, the Principal Principle entails that chance theories must assign themselves a chance of 1 . But this simple regularity account does not require chance theories to assign themselves a chance of 1 . Thus this simple regularity account is incompatible with the Principal Principle.

In order to avoid this worry, Hall (1994) and Lewis (1994) proposed a modified version of the Principal Principle, the New Principle:

$$
i c(A \mid T K)=c h_{T K}(A \mid T), \text { if defined. }
$$

The New Principle does not entail that $c h_{T K}(T)=1$, and thus has no difficulties accommodating regularity theories of chance.

Since the literature is split on which of these principles to adopt, we'll assess Callender and Cohen's account and Hoefer's account using both the Principal Principle and the New Principle. ${ }^{11}$

\subsection{Incomplete Chance Theories}

So far we've been taking the " $T$ " in " $c h_{T K}$ " to stand for a complete chance theory, a theory that encodes all of the chance facts that obtain at a world. And we've taken the Principal Principle and the New Principle to be formulated in terms of complete chance theories. But if there are multiple autonomous chance theories, then each theory encodes only a proper subset of the chance facts, and so is incomplete. Thus one might worry about whether the Principal Principle and New Principle can be applied to the autonomous chance theories Callender and Cohen (2009) and Hoefer (2007) discuss.

As it turns out, this is not a problem. One can use the fact that the Principal Principle and New Principle hold for complete chance theories to show that the assignments of incomplete chance theories will satisfy analogs of the Principal Principle and the New Principle.

We can see this as follows. Let an incomplete chance theory $I$ be a (non-trivial) disjunction of complete chance theories $T$. We can think of the content of $I$ as consisting of the chance facts that are entailed by all of the $T$ 's in this disjunction. We can then define a chance function $c h_{I K}$ for an incomplete chance theory $I$ as follows. Let $c h_{I K}(A)=x$ if all of the $T$ 's in I's disjunction are such that $c h_{T K}(A)=x$, and let $c h_{I K}(A)$ be undefined otherwise. So if we know that $I$ obtains and that $c h_{I K}(A)=x$, then we know that the chance of $A$ picked out by $K$ and the actual complete chance theory (whatever that may be) is $x$.

Likewise, let $c h_{I K}(A \mid B)=x$ if all of the T's in I's disjunction are such that $\operatorname{ch}_{T K}(A \mid B)=x$, and let $\operatorname{ch}_{I K}(A \mid B)$ be undefined otherwise. So if we know that $I$ obtains and that $\operatorname{ch}_{I K}(A \mid B)=x$,

\footnotetext{
${ }^{11}$ Some other ways of modifying the Principal Principle have also been proposed, such as the General Principle of Ismael (2009). But due to space restrictions, I'll restrict my attention to the Principal Principle and the New Principle.
} 
then we know that the chance of $A$ conditional on $B$ picked out by $K$ and the actual chance theory (whatever that may be) is $x .{ }^{12,13}$

Given these characterizations, one can use the Principal Principle to derive an analogous principle for incomplete chance theories: ${ }^{14}$

$$
i c(A \mid I K)=\operatorname{ch}_{I K}(A), \text { if defined. }
$$

Likewise, one can use the New Principle to derive an analogous principle for incomplete chance theories:

$$
i c(A \mid I K)=c h_{I K}(A \mid I), \text { if defined. }
$$

Thus, given this characterization of incomplete chance theories, we can harmlessly take the Principal Principle and the New Principle to hold for both complete and incomplete chance theories.

For convenience, I'll take $T$ to range over both complete and incomplete chance theories in what follows. Likewise, I'll use the term "chance theory" to stand for both complete and incomplete chance theories.

\subsection{Diverging Chance Assignments}

The accounts proposed by Callender and Cohen (2009) and Hoefer (2007) face worries about conflicts: if we allow multiple autonomous chance theories, then these theories could impose inconsistent constraints on rational belief. With this worry in mind, Callender and Cohen (2009) and Hoefer (2007) discuss several kinds of diverging chance assignments, and argue that they are not problematic. But while the cases of divergence they discuss are not problematic, there are other cases of divergence which are. To get clear about this, let us consider the ways in which a pair of chance assignments might diverge.

First, we might have a case where $c h_{T K}(A) \neq c h_{T K}(A B)$. This kind of divergence is not generally problematic, since $c h_{T K}(A)$ and $c h_{T K}(A B)$ constrain credences in different propositions.

\footnotetext{
${ }^{12}$ This is how we proceed if we start with an account like Lewis's, since his account begins by providing us with complete chance theories, and the task is to make sense of incomplete chance theories. Proponents of the Callender, Cohen and Hoefer's approaches have to proceed in the reverse direction: since their accounts begin by providing us with incomplete chance theories, their task is to make sense of complete chance theories. They can do so as follows. Let a complete chance theory $T$ at a world be a conjunction of all of the incomplete chance theories $I$ that obtain at that world. Then define a chance function $c h_{T K}$ for a complete chance theory $T$ as follows. Let $c h_{T K}(A)=x$ iff there is an $I$ in $T$ 's conjunction such that $c h_{I K}(A)=x$. And let $c h_{T K}(A \mid B)=x$ iff there is an $I$ in $T$ 's conjunction such that $c h_{I K}(A \mid B)=x$. (If the incomplete chance theories disagree in certain respects-if, for example, $I$ and $I^{*}$ obtain at the same world, and $c h_{I K}(A) \neq c h_{I^{*} K}(A)$ - then there won't be a well-defined complete chance function at that world.)

${ }^{13}$ This characterization of conditional chance for incomplete chance theories is slightly broader than the standard characterization of conditional probabilities. It will agree with the standard characterization (i.e., $\left.c h_{I K}(A \mid I)=c h_{I K}(A I) / c h_{I K}(I)\right)$ whenever the standard characterization is well-defined. But it will also be welldefined in some cases in which $c h_{I K}(A I) / c h_{I K}(I)$ is not. For example, suppose $I$ is a disjunction of $T_{1}$ (which assigns 0.1 and 0.2 to $A I$ and $I$ ) and $T_{2}$ (which assigns 0.2 and 0.4 to $A I$ and $I$ ). Then both $c h_{I K}(A I)$ and $\operatorname{ch}_{I K}(I)$ will be undefined. But $\operatorname{ch}_{I K}(A \mid I)$ will still be defined-it will be 0.5. (I've employed this alternative characterization because it is more appealing, but nothing in what follows hangs on this.)

${ }^{14}$ This derivation, and the analogous derivation for the New Principle, are given in the Appendix.
} 
For example, suppose $A$ is the proposition that the first of a sequence of coin tosses lands heads, and $B$ is the proposition that the second of this sequence of coin tosses lands heads. Suppose that $c h_{T K}(A)=1 / 2$ while $c h_{T K}(A B)=1 / 4$. Given the Principal Principle, a rational agent with total evidence $T K$ is required to have a credence of $1 / 2$ in $A$ and a credence of $1 / 4$ in $A B$. But these prescriptions are not in conflict.

Second, we might have a case where $c h_{T K}(A) \neq c h_{T K^{*}}(A)$. Again, this kind of divergence is not generally problematic, because $c h_{T K}(A)$ and $c h_{T K^{*}}(A)$ constrain different (conditional) credences. For example, let $A$ be as before, let $K$ be a description of the world before the coin toss, and let $K^{*}$ be a description of the world after the coin toss. Further suppose that $c h_{T K}(A)=1 / 2$ while $c h_{T K^{*}}(A)=1$. Given the Principal Principle, a rational agent is required to have an initial credence of $1 / 2$ in $A$ conditional on $T K$, and an initial credence of 1 in $A$ conditional on $T K^{*}$. But this is not problematic, since these prescriptions are not in conflict.

Third, we might have a case where $c h_{T K}(A) \neq c h_{T^{*} K}(A)$. Again, this kind of divergence is not usually problematic, because $c h_{T K}(A)$ and $c h_{T^{*} K}(A)$ constrain different (conditional) credences. For example, let $A$ be as before, and let $T$ and $T^{*}$ be two incompatible chance theories such that $c h_{T K}(A)=1 / 2$ while $c h_{T^{*} K}(A)=2 / 3 .{ }^{15}$ Given the Principal Principle, a rational agent is required to have an initial credence of $1 / 2$ in $A$ conditional on $T K$, and an initial credence of $2 / 3$ in $A$ conditional on $T^{*} K$. But this is unproblematic, since these prescriptions are not in conflict.

Let's look at a more concrete example. Suppose we know that animal psychology assigns the donkey a chance of 0.8 of moving up to eat a given bale of hay, while statistical mechanics assigns the donkey a chance of 0.801 of moving up to eat the hay. Given this, what should our credence be that the donkey will move up to eat the hay?

The answer will depend on how we flesh out the details. Suppose that both theories are assigning a chance to the same proposition $A$; i.e., that the outcome animal psychology assigns a chance to lines up exactly with the macrostate statistical mechanics assigns a chance to. And suppose that both chance assignments employ the same background information proposition $K$; i.e., that the initial set-up the animal psychology chance assignment employs lines up exactly with the initial macrostate the statistical mechanical chance assignment employs. ${ }^{16}$ Then we have an instance of a type 3 divergence, where $c h_{T K}(A) \neq c h_{T^{*} K}(A)$. And in the simple case in which an agent's total evidence is $T K \vee T^{*} K$, then her credence should be equal to the weighted average of these two chance assignments, where the weights are given by her credence that each theory is correct. That is, her credence in $A$ should be equal to her credence that animal psychology provides the correct chances multiplied by the chance animal psychology assigns $A$, plus her credence that statistical mechanics provides the correct chances multiplied by the chance statistical mechanics assigns $A .^{17}$

Although the first three cases of diverging chance assignments are not generally problematic,

\footnotetext{
${ }^{15}$ Incompatible in the sense that both cannot obtain at the same world-i.e., $T \Leftrightarrow \neg T^{*}$.

${ }^{16}$ Of course, in most cases the propositions these chances are assigned to and the background propositions these assignments employ will not be the same. For example, statistical mechanical background propositions generally require a specification of things like the number of particles in the system, while animal psychology background propositions generally do not.

${ }^{17}$ See Lewis (1986), p.106-108. For a more general characterization of what one's credences should be, given any way of filling in these details, see Meacham (2005), p.293.
} 
there are other cases of divergence which are problematic. Here is a fourth kind of divergence. Consider a case where $c h_{T K}(A) \neq c h_{T^{*} K^{*}}(A)$ and $T K \Leftrightarrow T^{*} K^{*}$. Given the Principal Principle, it follows that rational agents must be such that:

$$
\begin{aligned}
& i c(A \mid T K)=c h_{T K}(A)=x, \\
& i c\left(A \mid T^{*} K^{*}\right)=c h_{T^{*} K^{*}}(A) \neq x, \\
& i c(A \mid T K)=i c\left(A \mid T^{*} K^{*}\right),
\end{aligned}
$$

which is impossible. So if these kinds of chance assignments obtain, and we adopt the Principal Principle, then the chances will impose inconsistent constraints on rational belief.

Here is a fifth case of divergence, which also leads to problems. Consider a case where $c h_{T K}(A \mid T) \neq c h_{T^{*} K^{*}}\left(A \mid T^{*}\right)$ and $T K \Leftrightarrow T^{*} K^{*}$. Given the New Principle, it follows that rational agents must be such that:

$$
\begin{aligned}
& i c(A \mid T K)=\operatorname{ch}_{T K}(A \mid T)=x, \\
& i c\left(A \mid T^{*} K^{*}\right)=c h_{T^{*} K^{*}}\left(A \mid T^{*}\right) \neq x, \\
& i c(A \mid T K)=i c\left(A \mid T^{*} K^{*}\right),
\end{aligned}
$$

which is impossible. So if these kinds of chance assignments obtain, and we adopt the New Principle, then the chances impose inconsistent constraints on rational belief. ${ }^{18}$

So the Conflicts Problem concerns cases like the fourth and fifth types of divergences. If the accounts of Callender and Cohen (2009) and Hoefer (2007) allow for chance theories which diverge in these ways, then the chances provided by their accounts will impose inconsistent constraints on rational belief.

\section{Callender and Cohen's Account}

\subsection{The Account}

Callender and Cohen (2009) propose a variant of Lewis's (1994) account of laws and chances. As with Lewis's account, the idea is that the laws and chances are given by the description of the occurrent facts which best satisfies, on balance, the desiderata of simplicity and informativeness. And Callender and Cohen acknowledge that our assessment of how simple a given description is will depend on the language we use. Where they diverge from Lewis is that they do not go on to specify a special language to employ when evaluating the simplicity of these descriptions. Instead, they relativize the laws and chances to the vocabulary one employs.

At any given world, on this account, there are many different laws and chances, corresponding to the many different choices of vocabulary. For example, there are physical laws (which you get by using a vocabulary whose predicates correspond to those of physics), there are biological

\footnotetext{
${ }^{18}$ One can see the fourth and fifth types of divergence as instance of a single kind of divergence: cases where $T K \leftrightarrow T^{*} K^{*}$, and yet $T K$ and $T^{*} K^{*}$ pick out different chances.

One can also see the fourth and fifth types of divergence as a special instance of type 2 and type 3 divergences. (This is one of the reasons why the second and third divergences were described as not usually being problematic.)
} 
laws (which you get by using a vocabulary whose predicates correspond to those of biology), there are the laws of economics (which you get by using a vocabulary whose predicates correspond to those of economics), and so on.

Callender and Cohen's account neatly avoids the complaints they and Hoefer raise about Lewis's account. Unlike Lewis's account, it is not only the fundamental theory (presumably physics) that deals with laws and chances. The other sciences, including the special sciences, will provide laws and chances as well, and these laws and chances have the same metaphysical status as those of fundamental physics. Likewise, their account can recover the chances of ordinary life, like the chance of rain or the chance of a coin landing heads, regardless of what the chances of fundamental physics turn out to be. Given a vocabulary with terms for ordinary events of these kinds, Callender and Cohen's account will yield chances for these events.

Since different vocabularies will yield different laws and chances, Callender and Cohen's account allows a number of autonomous laws and chances to obtain at a world. The Conflicts Problem threatens: will these chances impose inconsistent constraints on rational belief?

\subsection{The 90 Particle World}

I'll suggest that Callendar and Cohen's account does lead to problematic conflicts. I'll do so by sketching a toy model of how a divergence of types 4 and 5 might plausibly arise given the account of laws and chances they propose.

Consider a world that begins with 90 particles, ten of which have 1 gram of mass, ten of which have 2 grams of mass, and so on, up to 9 grams of mass. These particles generally move about in accordance with the laws of classical mechanics. However, at one particular time, $\tau$, a number of these particles disappear, in a stochastic-looking fashion. Here is the distribution of how many particles of each mass survive (call this $S_{1}$ ):

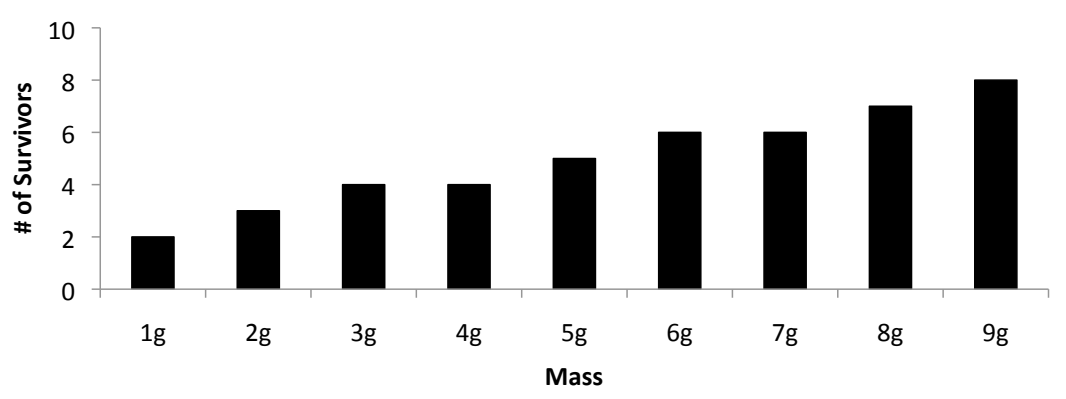

Now consider a vocabulary that employs the predicates of classical physics. Given such a vocabulary, the laws and chances on Callender and Cohen's account might be something like this (where I've divided the laws here into the non-chancy laws $(L)$ and the chancy laws $(T)$ ):

L: There are initially 10 particles of each integer of mass in grams between 1 and 9 , and nothing else, and (while they survive) these particles obey Newton's laws. ${ }^{19}$

\footnotetext{
${ }^{19}$ On Lewis's original account, a statement regarding the initial conditions like this would not have been a law, since
} 
T: The chance of a particle surviving after $\tau=\operatorname{mass}(\mathrm{g}) / 10$.

In this case, one might plausibly take $L$ and $S_{1}$ together to entail $T$ on Callender and Cohen's account, since they arguably specify enough about the world to fix what the chances (given this vocabulary) will be.

Now consider a second world, almost qualitatively identical to the first, where a slightly different distribution of particles of each mass survive the vanishing at $\tau$ (call this $S_{2}$ ):

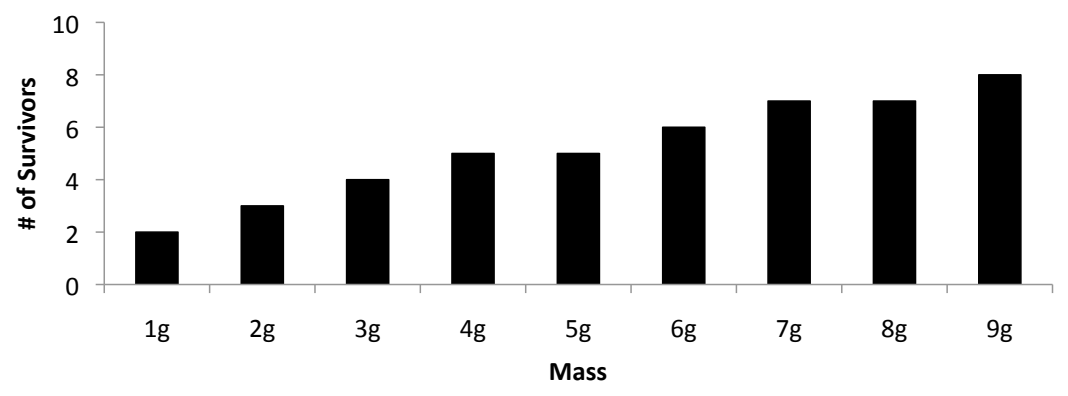

If we adopt a vocabulary that employs the predicates of classical physics, then the laws and chances on Callender and Cohen's account might plausibly be the same as before, $L$ and $T$. And, as before, one might plausibly take $L$ and $S_{2}$ to entail $T$ on Callender and Cohen's account.

Now let's consider a slightly different vocabulary one might employ. This vocabulary will employ many of the same predicates as before. But instead of using the mass predicate, it employs the schmass predicate, where:

An object has 2 schgrams of schmass iff it has 8 grams of mass,

An object has 4 schgrams of schmass iff it has 6 grams of mass,

An object has 6 schgrams of schmass iff it has 4 grams of mass,

An object has 8 schgrams of schmass iff it has 2 grams of mass,

Otherwise, an object has $x$ schgrams of schmass iff it has $x$ grams of mass.

So this is what the frequency of survivors described by $S_{1}$ will look like in terms of schmass:

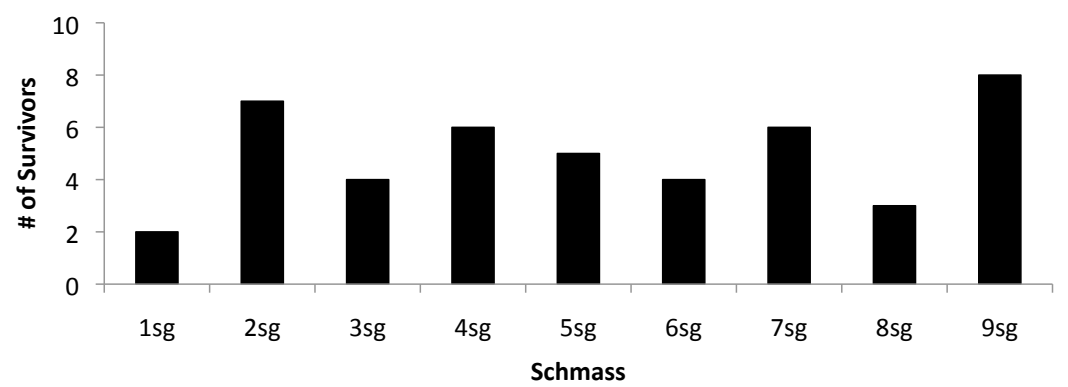

the laws are required to be regularities entailed by the axioms which, on balance, best satisfy the desiderata of simplicity, informativeness and fit, and statements about initial conditions are not regularities. But there are various reasons to drop this requirement (see Loewer (2001) and Winsberg (2008)). 
And here is what the frequency of survivors described by $S_{2}$ will look like in terms of schmass:

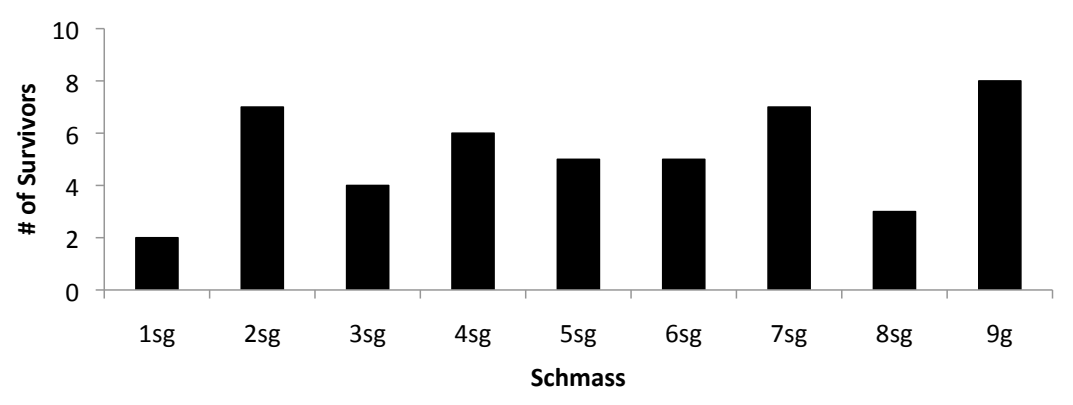

Given this vocabulary, the laws and chances on Callender and Cohen's account at both worlds might be something like this:

L: There are initially 10 particles of each integer of schmass in schgrams between 1 and 9 , and nothing else, and (while they survive) these particles obey Newton's laws. ${ }^{20}$

$\mathbf{T}^{*}$ : The chance of a particle surviving after $\tau=0.5 .^{21}$

And as before, one might plausibly take $L$ and $S_{1}$ to entail $T^{*}$ on Callender and Cohen's account, since they arguably specify enough about the world to fix what the chances (given this vocabulary) will be. Likewise, one might plausibly take $L$ and $S_{2}$ to entail $T^{*}$.

Let's pause for a moment to work out some chance assignments. Given $L$ and $T$, the chance of $S_{1}$ obtaining is $\approx 2.5 \cdot 10^{-6}$. And the chance of $S_{2}$ obtaining is $\approx 1.7 \cdot 10^{-6}$. So given $L$, $T$ and that either $S_{1}$ or $S_{2}$ will obtain, the chance of $S_{1}$ is $\approx \frac{2.5 \cdot 10^{-6}}{2.5 \cdot 10^{-6}+1.7 \cdot 10^{-6}} \approx 0.596$. That is, $\operatorname{ch} h_{T L\left(S_{1} \vee S_{2}\right)}\left(S_{1}\right) \approx 0.596$.

Given $L$ and $T^{*}$, on the other hand, the chance of $S_{1}$ obtaining is $\approx 1.4 \cdot 10^{-9}$, and the chance of $S_{2}$ obtaining is $\approx 6.1 \cdot 10^{-10}$. Thus given $L, T^{*}$ and $S_{1} \vee S_{2}$, the chance of $S_{1}$ is $\approx \frac{1.4 \cdot 10^{-9}}{1.4 \cdot 10^{-9}+6.1 \cdot 10^{-10}} \approx 0.697$. So $\operatorname{ch}_{T^{*} L\left(S_{1} \vee S_{2}\right)}\left(S_{1}\right) \approx 0.697$.

Given the Principal Principle, it follows from these chances that a rational initial credence function must be such that:

$$
i c\left(S_{1} \mid L\left(S_{1} \vee S_{2}\right)\right)=i c\left(S_{1} \mid T L\left(S_{1} \vee S_{2}\right)\right)=\operatorname{ch} h_{T L\left(S_{1} \vee S_{2}\right)}\left(S_{1}\right) \approx 0.596
$$

where the first step follows from the fact that both $L S_{1}$ and $L S_{2}$ entail $T$. It also follows from these chances that a rational initial credence function must be such that:

$$
i c\left(S_{1} \mid L\left(S_{1} \vee S_{2}\right)\right)=i c\left(S_{1} \mid T^{*} L\left(S_{1} \vee S_{2}\right)\right)=c h_{T^{*} L\left(S_{1} \vee S_{2}\right)}\left(S_{1}\right) \approx 0.697,
$$

\footnotetext{
${ }^{20}$ Of course, Newton's laws will have to be reformulated here in terms of schmass, and so will do worse with respect to simplicity in this vocabulary than in the vocabulary of classical physics. I'm assuming here that these laws will still do well enough with respect to informativeness to beat out any competitors.

${ }^{21}$ I.e., the chances might plausibly be given by $T^{*}$ because the average number of survivors is approximately 0.5 , and there arguably aren't any competing chances that would do better, given this vocabulary, with respect to simplicity and informativeness.
} 
where the first step follows from the fact that both $L S_{1}$ and $L S_{2}$ entail $T^{*}$. Together, (14) and (15) yield a type 4 divergence. Given the Principal Principle, these chances impose inconsistent constraints on rational belief.

Now let's look at how things go if we adopt the New Principle. First, note that:

$$
\operatorname{ch}_{T L\left(S_{1} \vee S_{2}\right)}\left(S_{1} \mid T\right)=\operatorname{ch}_{T L\left(S_{1} \vee S_{2}\right)}\left(S_{1}\right),
$$

because in this special case, $\operatorname{ch} h_{T\left(S_{1} \vee S_{2}\right)}(T)=1 .^{22}$ Likewise, note that:

$$
\operatorname{ch}_{T^{*} L\left(S_{1} \vee S_{2}\right)}\left(S_{1} \mid T^{*}\right)=\operatorname{ch}_{T^{*} L\left(S_{1} \vee S_{2}\right)}\left(S_{1}\right),
$$

because $c h_{T^{*} L\left(S_{1} \vee S_{2}\right)}\left(T^{*}\right)=1 .{ }^{23}$ Thus it follows from the New Principle that a rational initial credence function must be such that:

$$
i c\left(S_{1} \mid L\left(S_{1} \vee S_{2}\right)\right)=i c\left(S_{1} \mid T L\left(S_{1} \vee S_{2}\right)\right)=\operatorname{ch}_{T L\left(S_{1} \vee S_{2}\right)}\left(S_{1} \mid T\right)=\operatorname{ch}_{T L\left(S_{1} \vee S_{2}\right)}\left(S_{1}\right) \approx 0.596 .
$$

Likewise, it follows that:

$$
i c\left(S_{1} \mid L\left(S_{1} \vee S_{2}\right)\right)=i c\left(S_{1} \mid T^{*} L\left(S_{1} \vee S_{2}\right)\right)=c h_{T^{*} L\left(S_{1} \vee S_{2}\right)}\left(S_{1} \mid T^{*}\right)=c h_{T^{*} L\left(S_{1} \vee S_{2}\right)}\left(S_{1}\right) \approx 0.697 .
$$

Together, (18) and (19) yield a type 5 divergence. Given the New Principle, these chances impose inconsistent constraints on rational belief.

\section{Hoefer's Account}

\subsection{The Account}

Like Callender and Cohen, Hoefer (2007) proposes a modified version of Lewis's account. Like Lewis, Hoefer takes the chances to be given by the "best system", the description of the occurrent facts that best satisfies, on balance, the desiderata of simplicity and informativeness. But Hoefer's account diverges from Lewis's in some important ways.

First, Hoefer's account is an account of chance alone, not a combined account of laws and chances. On Lewis's account, the chances and laws are determined together. This makes what the chances are, and whether there are chances, dependent on what the laws are. But Hoefer wants his account to be able to recover ordinary chances, regardless of what the laws turn out to be. So Hoefer drops this feature of Lewis's account.

Second, Hoefer employs a broader understanding of simplicity and informativeness, according to which these desiderata take into account the usefulness of a candidate system for agents like ourselves. Thus simplicity selects for something like user-friendliness, taking comprehensibility and ease of use for agents like ourselves into consideration. Likewise, informativeness selects for something like helpfulness, taking utility and relevance to agents like ourselves into consideration.

\footnotetext{
${ }^{22}$ On regularity accounts, it generally won't be the case that $c h_{T K}(T)=1$. But it will be the case that $c h_{T K}(K)=1$. Thus $c h_{T L\left(S_{1} \vee S_{2}\right)}\left(L\left(S_{1} \vee S_{2}\right)\right)=1$. And since $L\left(S_{1} \vee S_{2}\right)$ entails $T$, it follows that $c h_{T L\left(S_{1} \vee S_{2}\right)}(T)=1$.

${ }^{23} \operatorname{ch}_{T^{*} L\left(S_{1} \vee S_{2}\right)}\left(L\left(S_{1} \vee S_{2}\right)\right)=1$, and since $L\left(S_{1} \vee S_{2}\right)$ entails $T^{*}$, it follows that $c h_{T^{*} L\left(S_{1} \vee S_{2}\right)}\left(T^{*}\right)=1$.
} 
This understanding frees Hoefer from having to pick a privileged vocabulary with which to assess candidate systems. Given Hoefer's understanding of simplicity and informativeness, we can assess each candidate system using whichever vocabulary shows them off to best advantage. We couldn't do this on Lewis's account, because this move leads to absurd results. Consider a language containing the predicate $W$, which applies to all and only things in the actual world. In this language, " $\forall x W x$ " offers a maximally simple and informative description of the world. ${ }^{24}$ This trivializes Lewis's account. But on Hoefer's account this problem won't arise, since trivial theories like the one just described won't count as simple and informative, given Hoefer's understanding of these desiderata.

This understanding also leads Hoefer to a different picture of what the best system will look like. Consider a candidate system that includes only the microphysical chances. And consider whether the system would be improved by adding an (incomplete) chance theory from the special sciences, which assigns chances that don't quite line up with the microphysical chances. Adding such a special science theory to a candidate system will increase its complexity. But if the special science theory is helpful and user-friendly enough — and deriving chances about this phenomena from the microphysical chances difficult enough — then the informativeness gained by adding such a theory will outweigh this increase in complexity. So we should expect the best system to consist of a number of autonomous chance theories, describing various kinds of phenomenaquantum mechanics, roulette wheels, rain, cancer rates, and so on.

A final difference between Hoefer's and Lewis's account shows up in some of Hoefer's earlier work. In Hoefer's earlier papers, he only allows chances to apply to propositions about "small-to-medium sized" events. ${ }^{25}$ And Hoefer maintained that it is the Principal Principle, not the New Principle, that correctly describes the link between credence and chance. ${ }^{26}$ In his more recent work, Hoefer adopts a position that is more similar to Lewis's. In particular, Hoefer allows chances to apply to "big" events, and proposes that we adopt something like the New Principle. ${ }^{27}$

\footnotetext{
${ }^{24}$ See Lewis (1983).

${ }^{25}$ See Hoefer (1997), Hoefer (2007). This feature of Hoefer's earlier account is controversial. One worry is that the restriction to propositions that are not "too big" requires chances to violate the probability axioms, since usual framework requires the set of propositions to which probabilities are assigned to be closed under conjunction and disjunction. Another worry is that the restriction to propositions that are not "too big" rules out some of the chances assigned by our actual scientific theories. A third point of contention is over whether we should abandon the New Principle, since some have argued that there are abstract reasons for favoring the New Principle over the Principal Principle (see Hall (2004)), though others have argued that adopting the New Principle violates the spirit of the regularity approach (see Hoefer (1997), Briggs (2009)). A fourth point of contention is over whether this restriction succeeds in avoiding undermining worries (see Briggs (2009)).

${ }^{26}$ In particular, Hoefer argues that the restriction to "small-to-medium sized" events trivializes the New Principle. Given Hoefer's restriction, $c h_{T K}(T)$ won't be well-defined, since $T$ encodes information about the entire distribution of occurrent facts, and is thus too big to be assigned a chance. Thus $c h_{T K}(A \mid T)$ won't be well-defined. So if we adopt the New Principle, the chances on the right-hand side of (5) will all be undefined, and the principle won't impose any constraints on credence. (Hoefer raises other complaints against the New Principle as well; see Hoefer (1997).) On the flip side, Hoefer argues that the restriction to "small-to-medium sized" events resolves the undermining worries that arise from applying the Principal Principle to regularity accounts of chance. Roughly, the idea is that any proposition informative enough to have undermining potential won't be assigned a well-defined chance; see Hoefer (1997).

${ }^{27}$ See Hoefer (forthcoming). Strictly speaking, Hoefer suggests that we adopt a version of the New Principle that is
} 
I'll assess both versions of Hoefer's account in section 4.2. First, I'll consider the account just described, which accepts Hoefer's earlier restriction on the domain of chance and adopts the Principal Principle. Then I'll consider Hoefer's later account which gives up this restriction and adopts the New Principle.

Like Callender and Cohen's account, Hoefer's account avoids the complaints that Callender, Cohen and Hoefer raise against Lewis's account of chance. Unlike Lewis's account, Hoefer's account allows for autonomous chances for the special sciences, since there are a number of distinct chance theories in the special sciences that would be useful to agents like us. Likewise, Hoefer's account is well-positioned to recover ordinary chances.

Since Hoefer's account appears to allow for multiple autonomous chance theories, the Conflicts Problem threatens. Will these chances place inconsistent constraints on rational belief?

\subsection{The $100 \times 100$ Grid World}

In what follows, I'll present a toy model for how divergences of type 4 and 5 might arise for an account like Hoefer's. In particular, I'll consider how problems might arise for two versions of Hoefer's account: the original account, which only allows chances to be assigned to propositions about small-to-medium sized events and adopts the Principal Principle, and a variant which does not restrict the domain of chance and adopts the New Principle. In each case, I'll use the toy model to show how problematic divergences might arise.

Consider a world that consists of a 100 by 100 grid of stationary objects. These objects are gray until time $\tau$. Then, at time $\tau$, approximately half of the objects turn white, and half of them turn black, in a stochastic-looking fashion. Of course, even though approximately half of them turn white, there will be some variation in how many of the objects turn white in each row of the grid. Let us suppose that in the first row of the grid, 40 objects turn white.

Given Hoefer's account, one chance theory that might plausibly obtain is this:

T: The chance of an object turning white at time $\tau$ is 0.5 .

These "micro chances" provide a simple and informative way for us to encode information about the frequencies of color transitions of the objects in the grid.

In addition to the frequencies of each object turning white, suppose that agents like ourselves are interested in certain macroscopic features of the grid's color distribution. In particular, suppose that we happen to care about the frequencies of white versus black-headed columns. Given Hoefer's account, here is another chance theory that might plausibly obtain:

$\mathbf{T}^{*}$ : The chance of a column becoming white-headed at $\tau$ is 0.4 .

These "macro chances" provide a simple and informative way for us to encode information about the frequencies of columns in the grid turning white-headed.

Let $K$ be a proposition describing all of the qualitative facts about the world except for the color of the first object in the last column of the grid after $\tau$. Since $K$ describes pretty much everything about the world, it plausibly allows us to deduce that both $T$ and $T^{*}$ obtain, given

defined over incomplete chance theories, such as (7). 
Hoefer's account. Let $A$ be the proposition that the first object in the last column of the grid turns white at $\tau$.

Consider Hoefer's original account, which adopts the Principal Principle. Since $K$ entails $T$, it follows from the Principal Principle that a rational initial credence function must be such that:

$$
i c(A \mid K)=i c(A \mid T K)=c h_{T K}(A)=0.5 .
$$

Since $K$ entails $T^{*}$, it also follows that a rational initial credence function must be such that:

$$
i c(A \mid K)=i c\left(A \mid T^{*} K\right)=\operatorname{ch}_{T^{*} K}(A)=0.4 \text {. }
$$

Since $T K \leftrightarrow T^{*} K$, (20) and (21) yield a type 4 divergence. So, given Hoefer's original account, there appear to be autonomous chance theories which impose inconsistent constraints on rational belief.

Now consider a variant of Hoefer's account, which allows chances to be assigned to "big" propositions and adopts the New Principle. Note that:

$$
\begin{aligned}
& \operatorname{ch}_{T K}(A \mid T)=\operatorname{ch}_{T K}(A), \\
& \operatorname{ch}_{T^{*} K}\left(A \mid T^{*}\right)=\operatorname{ch}_{T^{*} K}(A),
\end{aligned}
$$

since in this case $c h_{T K}(T)=1$ and $c h_{T^{*} K}\left(T^{*}\right)=1 .^{28}$ Thus it follows from the New Principle that a rational initial credence function must be such that:

$$
\begin{aligned}
& i c(A \mid K)=i c(A \mid T K)=c h_{T K}(A \mid T)=c h_{T K}(A)=0.5, \\
& i c(A \mid K)=i c\left(A \mid T^{*} K\right)=c h_{T^{*} K}\left(A \mid T^{*}\right)=c h_{T^{*} K}(A)=0.4 .
\end{aligned}
$$

$T K \leftrightarrow T^{*} K$, (24) and (25) yield a type 5 divergence. Thus, given this variant of Hoefer's account, there appear to be autonomous chance theories which impose inconsistent constraints on rational belief.

\section{Replies}

Both Callender and Cohen (2009) and Hoefer (2007) raise something like the Conflicts Problem, and offer replies to this worry. Their discussions focus on divergent chance assignments of types 1-3. And they rightly argue that these divergences are generally not problematic. It is less clear from their discussion how they would respond to divergences of types 4 or 5. In this section, I'll consider some potential responses.

The troublesome versions of the Conflicts Problem arise when, given the chances assigned by one's chance theory, one's chance-credence principle requires the very same conditional credence to take on different values. To avoid these inconsistent prescriptions, we need to either (i) modify our chance-credence principle so that it doesn't make these problematic prescriptions, or (ii) modify our account of chance so that it doesn't make these problematic chance assignments. Let's look at each of these options in turn.

\footnotetext{
${ }^{28}$ Since $c h_{T K}(K)=1$, and $K$ entails $T$, it follows that $c h_{T K}(K)=1$. Likewise, since $c h_{T K}(K)=1$, and $K$ entails $T$, it follows that $\operatorname{ch}_{T K}(T)=1$.
} 


\subsection{Modifying the Chance-Credence Principle}

The first way to get out of the Conflicts Problem is to modify the chance-credence principle so that it doesn't make problematic prescriptions. A response of this kind faces three challenges.

The first challenge is to spell out what exactly this new chance-credence principle is. Among other things, a satisfactory proposal should be precise, so that we can evaluate what exactly will happen in potential cases of conflict. ${ }^{29}$

The second challenge is to demonstrate that the new principle avoids inconsistent prescriptions when paired with one's chance theory. We want to ensure that this new principle will be bullet-proof - that there won't be "type 6 divergences" that yield inconsistent prescriptions given this new chance-credence principle. A satisfactory proposal should include a demonstration that such inconsistencies will not arise.

The third challenge is to address certain motivational worries facing this approach. One kind of motivational worry has to do with the chance-credence principle. A satisfactory proposal would ideally include a story about why this principle is plausible, and why it provides a better account of the chance-credence norm than the Principal Principle or the New Principle.

Another kind of motivational worry has to do with the chances one's chance theory posits. In particular, a motivational worry arises for those who, like Lewis, think that one of the definitive features of chance is that it constrains rational belief.

Consider a type 4 divergence, where $T K \leftrightarrow T^{*} K^{*}$ and $c h_{T K}(A)=x \neq c h_{T^{*} K^{*}}(A)=y$. In this case, given the Principal Principle, $T$ requires a rational agent to be such that $i c(A \mid T K)=$ $i c\left(A \mid T^{*} K^{*}\right)=x$, while $T^{*}$ requires a rational agent to be such that $i c(A \mid T K)=i c\left(A \mid T^{*} K^{*}\right)=y$. The new chance-credence principle will presumably ensure that these conflicting prescriptions don't arise. For example, it might tell the agent to just line up her priors with $c h_{T K}(A)$, and to ignore $c h_{T^{*} K^{*}}(A)$. But if so, then only $c h_{T K}(A)$ constrains rational belief. And if a definitive feature of chance is that it constrains rational belief, then by what right do we call $c h_{T^{*} K^{*}}(A)$ a chance?

This worry is especially pressing because there are other values which do tie to rational belief in a straightforward way. And if a definitive feature of chance is that it constrains rational belief, then one might argue that we should be calling these other values "chances".

In particular, suppose one succeeds in spelling out this new principle, and shows that it won't lead to inconsistencies. Then we can, by brute force, construct a single coherent function which encodes all of the prescriptions that these chances impose on us given this new principle. I.e., define $c h^{*}$ be a (partial) function which takes a pair of propositions and spits out a real number in the following way: $c h^{*}(A, G)$ equals $x$ if the new principle and the facts about chances together require $i c(A \mid G)=x$, and $c h^{*}(A, G)$ is undefined otherwise. Then $c h^{*}$ will constrain

\footnotetext{
${ }^{29}$ Particular issues arise here for those like Callender and Cohen (2009), who want to restrict the resources they appeal to. A prominent feature of Callender and Cohen's account is that it doesn't need to appeal to any privileged vocabulary or set of properties. This makes the construction of a conflict-avoiding chance-credence principle difficult. For example, consider a case like the 90 Particle World case, in which one physics-like vocabulary yields one set of chances, and a gruesome-physics-like vocabulary yields a conflicting set of chances. A modified chance-credence principle will need to adjudicate between these conflicting prescriptions. But since these two conflicting chance assignments are otherwise on a par, it's hard to see how to do so without effectively privileging one vocabulary over the other.
} 
rational belief in just the way that we would expect the chances to; i.e., in accordance with the Principal Principle.

So if we take a definitive feature of chance to be that it constrains rational belief, then this proposal faces a motivational worry. On the one hand, it looks like we shouldn't be calling all of the values the proposed chance theory assigns "chances", since they don't all constrain rational belief. On the other hand, we have a positive reason to call the values $c h^{*}$ assigns "chances", since they do. ${ }^{30}$

As an aside, it's worth mentioning why one tempting way of modifying the chance-credence principle in order to avoid problematic prescriptions-adding an admissibility clause to the Principal Principle/New Principle — doesn't look promising. ${ }^{31}$ This kind of proposal faces all three of the challenges sketched above-precisely characterizing the resulting principle (and the notion of admissibility it employs), showing that the resulting principle avoids conflicts, and addressing motivational questions regarding the principle and the chances it employs. But here, I'll restrict my attention to a some features of the third challenge - the challenge of motivating this modification of the chance-credence principle. ${ }^{32}$

Let's begin by considering the Principal Principle:

$$
i c(A \mid T K)=c h_{T K}(A), \text { if defined. }
$$

Why might one think we need to add an admissibility clause to this principle?

One potential worry is that the Principal Principle is too weak without an admissibility clause. For example, if $c h_{T K}(A)=x$, then the Principal Principle requires an agent to be such that $i c(A \mid T K)=x$. Thus, given conditionalization, the Principal Principle requires an agent whose total evidence is $T K$ to have a credence of $x$ in $A$. But one might worry that this kind of constraint is too weak. After all, it doesn't directly say anything about the credences of agents whose total evidence doesn't line up with $T K$. And realistic agents will never have total evidence like $T K$.

This worry is not compelling: although the Principal Principle doesn't directly impose constraints on realistic agents, it does impose indirect constraints on such agents. And once one takes this into account, one can see that the Principal Principle imposes all of the constraints on rational belief that one should want the chances to impose. There are a number of ways of defending this claim, but I'll only sketch one of them here. To simplify, let's put aside worries about undermining and focus on the Principal Principle.

Consider all of the $T K \mathrm{~s}$ that yield chance assignments. For any given $T K$, there will generally be lots of other $T K$ s nested inside it (those $T K$ s that strictly imply it), and lots of $T K$ s which

\footnotetext{
${ }^{30}$ Similar worries are raised regarding the New Principle by Arntzenius and Hall (2003).

${ }^{31}$ That is, adding an admissibility clause to Lewis's second formulation of the Principal Principle, (3), the formulation we've been employing here. Lewis's first formulation of the Principal Principle already includes an admissibility clause, an addition which is required in order to make the two formulations equivalent (see footnote 8). But the notion of admissibility employed by Lewis's first principle cannot be the notion of admissibility employed here, since that notion is of no help in resolving the Conflicts Problem-one can derive the same conflicting prescriptions in type 4 divergences using Lewis's first formulation of the Principal Principle, admissibility clause and all.

${ }^{32}$ There are various ways to spell out why the Lewis's second formulation of the Principal Principle and the New Principle don't require an admissibility clause; for example, see Hall (1994), Arntzenius (1995), Hall (2004), Meacham (2005), Nelson (2009) and Meacham (2010). One can see what follows as excerpts from these discussions.
} 
contain it (those $T K \mathrm{~s}$ which it strictly implies). (For example, if Ks are histories up to a time, a given $T K$ will contain $T K$ s whose histories run up to some future time, and will be contained within $T K$ s whose histories run up to some earlier time.) And there will generally be a locally largest $T K$ that isn't nested inside any other $T K$, and many locally smallest $T K \mathrm{~s}$ in which no other $T K \mathrm{~s}$ are nested. (For example, if Ks are histories, the largest $T K$ might pick out the initial state of the world, and the smallest $T K$ s complete histories.)

We can think of the constraints the Principal Principle imposes as telling us how our priors in these large $T K$ s should be divided among the smaller $T K$ s it contains (see Meacham (2005)). This constraint leaves two things open: how our prior in all possibilities should get divided up among the large $T K \mathrm{~s}$, and how our prior in the smallest $T K$ s should get divided up among individual worlds. But once we've fixed those two things, the Principal Principle fixes everything else.

To show that the Principal Principle is too weak, one must show that the correct chancecredence principle will impose more constraints on priors than the Principal Principle does. And there are only two places where additional constraints could be imposed. First, it could constrain how our prior in all worlds gets divided up among the large $T K \mathrm{~s}$. (This might fix things like one's prior in the GRW interpretation of quantum mechanics, and one's prior in particular initial wave functions.) But this is implausible-how we divide our initial credence among these large $T K \mathrm{~s}$ is beyond the scope of chance. Second, it could constrain how our initial credence in the smallest $T K$ s gets divided up among individual possible worlds. (Given classical statistical mechanics, this might constraint how one's prior in complete particle position-and-velocity histories gets divided up among worlds with different kinds of non-supervening mental states (like Chalmersstyle qualia), non-spatiotemporally located spirits, and so on.) Again, this is implausible, since these are precisely the kinds of things that the chances don't say anything about. So it doesn't seem like there's room for additional constraints on priors over and above those the Principal Principle imposes.

Thus the challenge facing anyone who thinks we need a principle stronger than the Principal Principle is this: (a) identify which of these two places additional constraints on priors should be imposed, and (b) show why, despite appearances, it's plausible to think that the chances should constrain our priors in these ways.

That said, for our purposes, we can put this issue aside. Even if it were the case that the Principal Principle needed to be strengthened, this wouldn't help with the Conflicts Problem. If the Principal Principle yields inconsistent prescriptions, then any stronger version of the principle will also yield inconsistent prescriptions.

The other potential worry one might have is that the Principal Principle is too strong without an admissibility clause. One might argue that there are cases where $c h_{T K}(A)=x$, an agent's total evidence is $T K$, and yet her credence in $A$ should not be $x$. In particular, one might think that in "crystal ball" cases, where (say) an agent gets evidence about the outcomes of future events, her credences should not line up with the chances.

If this "crystal ball evidence" is evidence over and above $T K$, then these cases can't pose a problem for the Principal Principle. The Principal Principle only tells agents to line up their credences with $c h_{T K}(A)$ if their total evidence is $T K$. So if an agent has crystal ball evidence $E$ in addition to $T K$, then the Principal Principle will not tell them to line up their credences with 
$c h_{T K}(A)$. To raise a problem for the Principal Principle, $T K$ needs to already include the crystal ball evidence. So let's restrict our attention to these kinds of cases.

There are a number of details here that remain to be fleshed out-how the crystal ball works, what kind of evidence about the crystal ball $T K$ includes, and so on. But we can bracket most of these details. Suppose that the crystal ball infallibly indicates that $A$ will occur (where $T K$ may or may not include details regarding how the crystal ball works, that it's infallible, and so on). Then either the agent's total evidence $T K$ entails $A$, or it doesn't. If $T K$ doesn't entail $A$, then the agent shouldn't heed the crystal ball's predictions, since her total evidence doesn't give her reason to think the crystal ball's predictions are correct. So she should line up her credences with the chances, just as the Principal Principle says. If $T K$ does entail $A$, then the agent clearly should have a credence of 1 in $A$. But if $T K$ entails $A$, then $c h_{T K}(A)=1$. So again, the agent should line up her credences with the chances, just as the Principal Principle says. Either way, we don't need admissibility to handle crystal ball cases.

One might resist this treatment of crystal ball cases by challenging the assumption that if $T K$ entails $A$, then $\operatorname{ch}_{T K}(A)=1$. Many Humeans will maintain that this claim is false, since they'll maintain that $c h_{T K}(T) \neq 1$ even thought $T K$ entails $T$. In particular, while they'll agree that if $K$ entails $A$ then $c h_{T K}(A)=1$, they'll disagree that if $T$ entails $A$ then $c h_{T K}(A)=1$. And thus they'll disagree that if $T K$ entails $A$ then $c h_{T K}(A)=1 .^{33}$

But this move does not support the claim that we need to appeal to admissibility. If one thinks that $c h_{T K}(T) \neq 1$, then one should adopt the New Principle, not the Principal Principle. And once we adopt the New Principle, we'll again get the right prescriptions in crystal ball cases, even if we follow the Humean in rejecting the claim that $c h_{T K}(A)$ must be 1 when $T K$ entails $A$.

(To see this, consider the crystal ball cases in which $T K$ entails $A$. It follows from the New Principle that:

$$
\begin{aligned}
i c(A \mid T K) & =c h_{T K}(A \mid T) \\
& =\frac{c h_{T K}(T A)}{c h_{T K}(T)} \\
& \geq \frac{c h_{T K}(K T)}{c h_{T K}(T)} \\
& =\frac{c h_{T K}(T)}{c h_{T K}(T)} \\
& =1,
\end{aligned}
$$

where the third step follows from the fact that since $T K$ entails $T A$, the probability axioms require $c h_{T K}(T A) \geq c h_{T K}(K T)$, and the fourth step follows from the fact that $c h_{T K}(K)=1$. So in crystal ball cases in which $T K$ entails $A$, the New Principle will prescribe the right credencesit will tell the agent to have a credence of 1 in $A$. Thus, again, we find that we don't need admissibility to handle crystal ball cases.)

\footnotetext{
${ }^{33}$ Likewise, crystal ball cases aside, a Humean might take this to be a reason to think the Principal Principle is too strong, since in cases where $c h_{T K}(T)=x<1$, an agent with total evidence $T K$ shouldn't have a credence of $x$ in $T$, even though this is what the Principal Principle requires. But, as Hall (1994) and Lewis (1994) show, this motivates a move to the New Principle, not the addition of an admissibility clause. And adopting the New Principle doesn't help with the Conflicts Problem.
} 
So although introducing an admissibility clause is a tempting way to try to avoid the Conflicts Problem, this approach isn't promising. In order to motivate the appeal to admissibility in a way that will help with the Conflicts Problem, one must establish that the Principal Principle and New Principle are too strong. And the main reason that has been offered for thinking that these principles are too strong — that they make the wrong prescriptions in "crystal ball cases"doesn't hold up to scrutiny.

\subsection{Modifying the Account of Chance}

The second way to get out of the Conflicts Problem is to modify one's account of chance so that it doesn't make problematic chance assignments. In particular, one can modify one's account by adding the requirement that all incomplete chance theories "line up" in a way which avoids conflicts.

How attractive is this option? It depends on one's account of chance.

Given Hoefer's account, this modification is easy to make. On Hoefer's account, what the chances are depends on our overall assessment of a package of these chance theories. One evaluates various packages of chance theories with respect to their combined simplicity and informativeness, and picks the package that does the best. This makes it easy to incorporate something like the above constraint. One can just add the requirement that the chance theories fit together in the appropriate way to the desiderata used to assess packages of theories, and then proceed as before.

Of course, this is to give up on autonomous chances-if we require the various chance theories to be compatible with one another, they're no longer autonomous. But it's not clear how important autonomous chances are to Hoefer's overall project. And given that this move offers a straightforward way of avoiding the Conflicts Problem, this seems a small price to pay.

Given Callender and Cohen's account, this move is less natural. On their account, the chances associated with each choice of vocabulary are determined separately. In order to adopt something like the above restriction, this would need to change-the chances associated with each vocabulary would need to be determined together, in order to ensure consistency.

Moreover, various issues arise when we consider how to add such a constraint to Callender and Cohen's account. Suppose that the most natural chance theory given vocabulary $V_{1}$ is $c h_{1}$, that the most natural chance theory given vocabulary $V_{2}$ is $c h_{2}$, and that these two chance assignments conflict. How do we resolve this conflict? One options is to declare that one or the other of these chance theories trumps the other. But such a decision would seem to privilege one of these vocabularies over the other. And to do so would be to give up the most prominent features of their account.

Another option would be to mimic Lewis's response to the possibility of ties with respect to best systems. Lewis suggests that if two candidate systems of laws do equally well, then we should take the laws to be all and only the laws that are common to both systems. ${ }^{34}$ Similarly, one might suggest that in cases of conflict, one should take the chances to be all and only those assignments that the don't conflict with the assignments of another theory. But this move is

\footnotetext{
${ }^{34}$ See Lewis (1983).
} 
problematic. For virtually any chance assignment, there will be some choice of vocabulary that yields a conflicting chance assignment. And eliminating every chance that conflicts with the assignment of some other chance theory threatens to eliminate chances entirely.

Finally, as we noted earlier, adopting such a requirement requires giving up the idea that these different chance theories can be autonomous. And this is an unhappy consequence for Callender and Cohen, since they take this to be an important feature of their account. ${ }^{35}$ So while this way of getting around the Conflicts Problem seems promising for proponents of Hoefer's account, it does not look as promising for proponents of Callender and Cohen's account.

\section{Conclusion}

Callender and Cohen (2009) and Hoefer (2007) propose versions of a regularity account of chance that allow multiple autonomous incomplete chance theories to obtain at a world. As we've seen, however, the demand that chance constrain rational belief is in tension with the demand for autonomous chances. If chances constrain rational belief, then the chances must line up with each other in order to avoid conflicting constraints. But if we require the chances to line up with each other, then they're not autonomous. So insofar as we want to hold on to the demand that chance constrains rational belief, we should give up on the demand for autonomous chances. ${ }^{36}$

\section{Appendix}

We can see that the analog of the Principal Principle holds for incomplete chance theories as follows. Suppose $c h_{I K}(A)=x$. Given the Principal Principle and the characterization of the incomplete chance function given in section 2.2, it follows that:

$$
\begin{aligned}
i c(A \mid I K) & =\frac{i c(A I K)}{i c(I K)}=\frac{\sum_{i} i c\left(A I T_{i} K\right)}{\sum_{i} i c\left(I T_{i} K\right)}=\frac{\sum_{i} i c\left(A I T_{i} K\right)}{\sum_{i} i c\left(T_{i} K\right)}=\frac{\sum_{i} i c\left(A \mid T_{i} K\right) \cdot i c\left(T_{i} K\right)}{\sum_{i} i c\left(T_{i} K\right)} \\
& =\frac{\sum_{i} c h_{T_{i} K}(A) \cdot i c\left(T_{i} K\right)}{\sum_{i} i c\left(T_{i} K\right)}=\frac{\sum_{i} x \cdot i c\left(T_{i} K\right)}{\sum_{i} i c\left(T_{i} K\right)}=x=c h_{I K}(A),
\end{aligned}
$$

where $i$ ranges over the complete chance theories $T_{i}$ in $I$ 's disjunction.

In a similar fashion, we can see that the analog of the New Principle holds for incomplete chance theories. Suppose $c h_{I K}(A \mid T)=x$. Given the New Principle and the characterization of incomplete chance function given in section 2.2, it follows that:

$$
\begin{aligned}
i c(A \mid I K) & =\frac{i c(A I K)}{i c(I K)}=\frac{\sum_{i} i c\left(A I T_{i} K\right)}{\sum_{i} i c\left(I T_{i} K\right)}=\frac{\sum_{i} i c\left(A I T_{i} K\right)}{\sum_{i} i c\left(T_{i} K\right)}=\frac{\sum_{i} i c\left(A \mid T_{i} K\right) \cdot i c\left(T_{i} K\right)}{\sum_{i} i c\left(T_{i} K\right)} \\
& =\frac{\sum_{i} c h_{T_{i} K}\left(A \mid T_{i}\right) \cdot i c\left(T_{i} K\right)}{\sum_{i} i c\left(T_{i} K\right)}=\frac{\sum_{i} x \cdot i c\left(T_{i} K\right)}{\sum_{i} i c\left(T_{i} K\right)}=x=c h_{I K}(A \mid I),
\end{aligned}
$$

\footnotetext{
${ }^{35}$ See Callender and Cohen (2010).

${ }^{36}$ Thanks to Craig Callender, Jonathan Cohen, Maya Eddon, Carl Hoefer, Alastair Wilson, members of the Monash Chance Conference, and two readers from Oxford University Press, for helpful comments and discussion. Special thanks to Carl Hoefer for a lengthy and illuminating correspondence on these issues.
} 
where $i$ ranges over the complete chance theories $T_{i}$ in $I$ 's disjunction.

\section{References}

Arntzenius, Frank. 1995. "Chance and the Principal Principle: Things Ain't What They Used To Be.” Unpublished Manuscript.

Arntzenius, Frank and Ned Hall. 2003. "On What We Know About Chance." British Journal for the Philosophy of Science 54:171-179.

Briggs, Rachael. 2009. “The Anatomy of the Big Bad Bug.” Noûs 43(3):428-449.

Callender, Craig and Jonathan Cohen. 2009. "A Better Best System Account of Lawhood." Philosophical Studies 145:1-34.

Callender, Craig and Jonathan Cohen. 2010. "Special Sciences, Conspiracy and the Better Best System Account of Lawhood." Erkenntnis 73:427-447.

Dunn, Jeff. 2011. "Fried Eggs, Theromdynamics, and the Special Sciences." British Journal for the Philosophy of Science 62:71-98.

Glynn, Luke. 2010. "Deterministic Chance." British Journal for the Philosophy of Science 61:51-80.

Hall, Ned. 1994. "Correcting the Guide to Objective Chance.” Mind 103:505-517.

Hall, Ned. 2004. "Two Mistakes About Credence and Chance." Australasian Journal of Philosophy 82:93-111.

Handfield, Toby and Alastair Wilson. 2012. Chance and Context. In Asymmetries in Time and Chance. Oxford University Press.

Hoefer, Carl. 1997. “On Lewis's Objective Chance: "Humean Supervenience Debugged”." Mind 106(422):321-334.

Hoefer, Carl. 2007. "The Third Way on Objective Probability: A Skeptic's Guide to Objective Chance." Mind 116:549-596.

Hoefer, Carl. forthcoming. Chance.

Ismael, Jenann. 2009. “Raid! Dissolving the Big, Bad Bug.” Nous 42:292-307.

Lewis, David. 1983. "New Work for a Theory of Universals." Australasian Journal of Philosophy 61:343-377.

Lewis, David. 1986. A Subjectivist's Guide to Objective Chance. In Philosophical Papers, Vol. 2. Oxford University Press pp. 83-132. 
Lewis, David. 1994. "Humean Supervenience Debugged.” Mind 103:473-490.

Loewer, Barry. 2001. "Determinism and Chance." Studies in the History of Modern Physics 32:609-620.

Meacham, Christopher J G. 2005. "Three Proposals Regarding a Theory of Chance.” Philosophical Perspectives 19:281-307.

Meacham, Christopher J G. 2010. "Two Mistakes Regarding the Principal Principle.” British Journal for the Philosophy of Science 61:407-431.

Nelson, Kevin. 2009. “On Background: Using Two-Argument Chance.” Synthese 1:165-186.

Schrenk, M. 2008. Selected Papers Contributed to the Sections of GAP.6, 6th International Congress of the Society for Analytical Philosophy. In Readings on Laws of Nature, ed. K. Dreimann H. Bohse and S. Walter. Mentis.

Taylor, Barry. 1993. "On natural properties in metaphysics.” Mind 102:81-100.

Winsberg, Eric. 2008. "Laws and Chances in Statistical Mechanics." Studies in History and Philosophy of Modern Physics 39:872-888. 